\title{
In Vitro Demonstration of Focused Ultrasound Thrombolysis Using Bifrequency Excitation
}

\author{
Izella Saletes, ${ }^{1,2}$ Bruno Gilles, ${ }^{1,2}$ Vincent Auboiroux, ${ }^{3,4}$ Nadia Bendridi, ${ }^{1}$ \\ Rares Salomir, ${ }^{3,5,6}$ and Jean-Christophe Béra ${ }^{1,2}$ \\ ${ }^{1}$ Inserm, U1032, LabTau and Université de Lyon, 69003 Lyon, France \\ ${ }^{2}$ Université Lyon 1, 69003 Lyon, France \\ ${ }^{3}$ Faculty of Medicine, University of Geneva, 1211 Geneva, Switzerland \\ ${ }^{4}$ Clinatec/LETI/CEA, 38054 Grenoble, France \\ ${ }^{5}$ Radiology Department, University Hospitals of Geneva, 1211 Geneva, Switzerland \\ ${ }^{6}$ Inserm, ADR 05 Rhône-Alpes, Auvergne, 69500 Bron, France \\ Correspondence should be addressed to Bruno Gilles; bruno.gilles@inserm.fr
}

Received 17 March 2014; Revised 5 June 2014; Accepted 25 June 2014; Published 27 August 2014

Academic Editor: Rajiv Chopra

Copyright ( 2014 Izella Saletes et al. This is an open access article distributed under the Creative Commons Attribution License, which permits unrestricted use, distribution, and reproduction in any medium, provided the original work is properly cited.

Focused ultrasound involving inertial cavitation has been shown to be an efficient method to induce thrombolysis without any pharmacological agent. However, further investigation of the mechanisms involved and further optimization of the process are still required. The present work aims at studying the relevance of a bifrequency excitation compared to a classical monofrequency excitation to achieve thrombolysis without any pharmacological agent. In vitro human blood clots were placed at the focus of a piezoelectric transducer. Efficiency of the thrombolysis was assessed by weighing each clot before and after sonication. The efficiencies of mono- $(550 \mathrm{kHz}$ ) and bifrequency ( 535 and $565 \mathrm{kHz}$ ) excitations were compared for peak power ranging from $70 \mathrm{~W}$ to $220 \mathrm{~W}$. The thrombolysis efficiency appears to be correlated to the inertial cavitation activity quantified by passive acoustic listening. In the conditions of the experiment, the power needed to achieve $80 \%$ of thrombolysis with a monofrequency excitation is reduced by the half with a bifrequency excitation. The thermal effects of bifrequency and monofrequency excitations, studied using MR thermometry measurements in turkey muscle samples where no cavitation occurred, did not show any difference between both types of excitations when using the same power level.

\section{Introduction}

Blood clot formation is a natural mechanism to prevent and stop bleeding, but it may lead to many vascular diseases like deep vein thrombosis or pulmonary embolism. Currently, thrombosis treatments correspond to two types of approaches that can be combined: surgical and other interventional techniques on one hand and drug based methods on the other hand [1-3]. Surgical methods are strongly invasive and not usable for patients who are usually already weak. Endovascular techniques are less invasive, but they nevertheless require adequate technical centres with qualified staff. In both cases the setting up of the treatment is long and may induce revascularization delays potentially associated with irreversible lesions on tissue. Thrombolytic drugs are usually used in the treatment of thrombosis: thanks to their large availability and their ease of use, they have greatly enhanced treatments of vascular diseases, but they still present a number of major drawbacks [4]: due to their nonselective action when they are injected in general bloodstream, they carry a significant haemorrhagic risk, and their administration is restricted by a lot of contraindications. Recent thrombolysis methods were proposed to combine thrombolytic agents with percutaneous techniques in order to get more selective treatments $[5,6]$, but haemorrhagic risks remain and constraints related to catheterization still require adequate technical centres.

Considering the limitations of these treatments, the use of ultrasound for thrombolysis has been foreseen as a promising 
technique especially when combined with fibrinolytic agents [7]. Ultrasound can be applied either using a catheter to locally sonicate blood clots [8-10] or externally. Catheterbased techniques enable rapid clot lysis, but they have the drawbacks of interventional techniques mentioned above. In the hope of reducing invasiveness, many studies focused on the use of transcutaneous ultrasound. In particular, a number of works have shown that low-intensity ultrasound was able to increase the activity of fibrinolytic agents [11-16], which enables reducing the drug quantity required for thrombolysis. Nevertheless, haemorrhagic risk associated with the use of thrombolytic drugs is still present, and resisting clots were also observed [17]. Transcutaneous ultrasound thrombolysis could be also achieved without any pharmacological agent using focused shock waves or high intensity ultrasound [18, 19] and recently received increasing attention with studies showing that transcutaneous focused ultrasound can achieve clot lysis within minutes both in vitro and in vivo [20-23]. In those works, cavitation appeared to play a major role in clot disruption, even if mechanisms involved in the process still have to be clarified.

Stimulation of the inertial cavitation activity can be achieved using a focused ultrasound excitation including two neighbouring frequencies instead of a monofrequency wave: this type of excitation enables, in some configurations, lowering of the inertial cavitation threshold [24] and an increase in the cavitation activity on a target of controlled roughness [25].

Such a stimulation of the cavitation activity could be an advantage for ultrasound thrombolysis, but because of higher pressure amplitudes involved for a bifrequency excitation at a given intensity, increased heating of tissue due to nonlinear propagation effects could also occur [26], which would be a drawback of the method. The present study aims at comparing mono- and bifrequency excitations both in terms of thrombolytic efficiency for an in vitro blood clot model and in terms of heating of tissue in the focal region. In a first set of experiments, passive ultrasound monitoring of the cavitation activity during thrombolysis is processed, and in a second set of experiments, $\mathrm{MR}$ imaging is used to characterize the temperature rise caused by both types of excitation and to dynamically visualize the clot disruption process. A single sonication configuration is used, with peak amplitudes comparable to the one successfully used by Maxwell et al. [20] to achieve ultrasound thrombolysis, but with increased duty cycle in order to achieve a better characterization of the thermal effects in the case of a bifrequency excitation at high exposure levels, when compared to a monofrequency excitation.

\section{Materials and Methods}

2.1. Ultrasound Generation. Acoustic excitations were generated by a focused spherical piezoelectric transducer (focal length: $100 \mathrm{~mm}$, aperture diameter: $100 \mathrm{~mm}$ ). The resonant frequency of the whole emission line, including a generator (AFG3102, Tektronix, USA), a power amplifier (1000 W, 0.1-6 MHz, Adece, France), and the transducer (Imasonic,
France), was $550 \mathrm{kHz}$. The $-3 \mathrm{~dB}$ focal volume at the resonant frequency was a $20 \mathrm{~mm}$ long and $3 \mathrm{~mm}$ wide ellipsoid. The transducer efficiency at the resonant frequency, measured using a radiation force balance method, was $84 \%$.

Two types of pulsed-wave excitation were used, according to $[25]$ :

(i) a monofrequency excitation which consisted in a pulsed-wave signal of a pure sine wave at frequency $f_{0}=550 \mathrm{kHz}$,

(ii) a bifrequency excitation which consisted in a pulsedwave signal made of the sum of two sine waves of slightly different frequencies, $f_{1}=535 \mathrm{kHz}$ and $f_{2}=$ $565 \mathrm{kHz}$, and of the same pressure amplitude.

In both cases, the pulse duration was $27 \mathrm{~ms}$, the duty cycle was $1: 10$, and the total treatment duration was 5 minutes. The exposure level was expressed in terms of the acoustic power that ranged from $P_{\mathrm{ac}}=70 \mathrm{~W}$ to $P_{\mathrm{ac}}=220 \mathrm{~W}$ for both types of excitation. In order to characterize the peak negative and positive pressures obtained in that range of excitation level, and the corresponding spatial-peak pulse-average intensities, a calibration was done using a hydrophone (Müller-Platte Needle Probe, Müller Instruments, Germany) placed at the focal point of the transducer in the absence of any clot. In order to avoid cavitation at high excitation levels, shorter pulses were used for this calibration process: $20 \mu$ s pulses for monofrequency excitation and $70 \mu$ s pulses for bifrequency excitation. Results are given in Table 1.

Examples of pressure waveforms measured at the focal point of the transducer are shown in Figure 1 at a power $P_{\mathrm{ac}}=$ $25 \mathrm{~W}$, for which no cavitation occurred, and at $P_{\mathrm{ac}}=165 \mathrm{~W}$ using a short pulse to avoid cavitation at this exposure level. It has to be noticed that, at a given power, the peak pressure of a bifrequency signal is $\sqrt{2}$ times higher than the one of a monofrequency signal, as can be seen on peak pressures of the signals in Figure 1.

A duty cycle as high as 1:10 was chosen to stimulate heating in the focal region, in order to characterize, as precisely as possible, the spatial distribution of the bifrequency pressure field in tissue at high amplitudes, and to compare temperature rise for both types of excitation at a given power. This aimed at evaluating the nonlinear propagation effects associated with the bifrequency waveforms by measuring the nonlinear increase in temperature rise-compared to a monofrequency wave of the same power-due to the higher peak pressure being involved in the bifrequency waveforms.

\subsection{Thrombolysis with Passive Listening of Inertial Cavitation} Activity. The thrombolysis experiments were carried out in a double-tank sketched in Figure 2 . The outer tank $(520 \times 270 \times$ $230 \mathrm{~mm}(L \times W \times H))$ was filled with degassed filtered water. To enable good transmission and avoid acoustic reflections, two acoustic windows were pierced in the inner tank $(200 \times 200 \times$ $215 \mathrm{~mm}(L \times W \times H))$, and an acoustic absorber consisting of a paraffin block was placed at the back of the outer tank. The blood clot samples (cf. below) were placed in PVC tubes (ID = $10 \mathrm{~mm}, \mathrm{OD}=12 \mathrm{~mm}$, and $H=15 \mathrm{~mm}$ ) at the focus of the transducer. In order to prevent cavitation occurring on the 
TABLE 1: Peak negative and positive pressures and pulse-average intensities measured at the focal point of the transducer in water for the range of exposure level used in the experiments and for both types of excitations.

\begin{tabular}{lccc}
\hline & Peak pressures & Spatial-peak pulse-average intensity & Total acoustic power \\
$p^{-}(\mathrm{MPa})$ & $p^{+}(\mathrm{MPa})$ & $I_{\text {sppa }}\left(\mathrm{W} / \mathrm{cm}^{2}\right)^{\mathrm{a}}$ & $P_{\mathrm{ac}}(\mathrm{W})$ \\
\hline & & Monofrequency excitation & 50 \\
4.2 & 3.6 & 360 & 110 \\
5.2 & 6.2 & 770 & 165 \\
5.5 & 8 & 1200 & 220 \\
\hline & 9.0 & - & 70 \\
5 & & Bifrequency excitation & 110 \\
6 & 7 & 510 & 750 \\
7.5 & 9 & $\sim 1000^{\mathrm{a}}$ & 150 \\
\hline
\end{tabular}

${ }^{a}$ Intensities are estimated by integrating waveforms recorded at the focal point. At $P_{\mathrm{ac}}=220 \mathrm{~W}$, occurrence of cavitation prevents from giving an accurate estimation of the intensity. Bifrequency waveform at $P_{\mathrm{ac}}=150 \mathrm{~W}$ was also perturbed by cavitation.

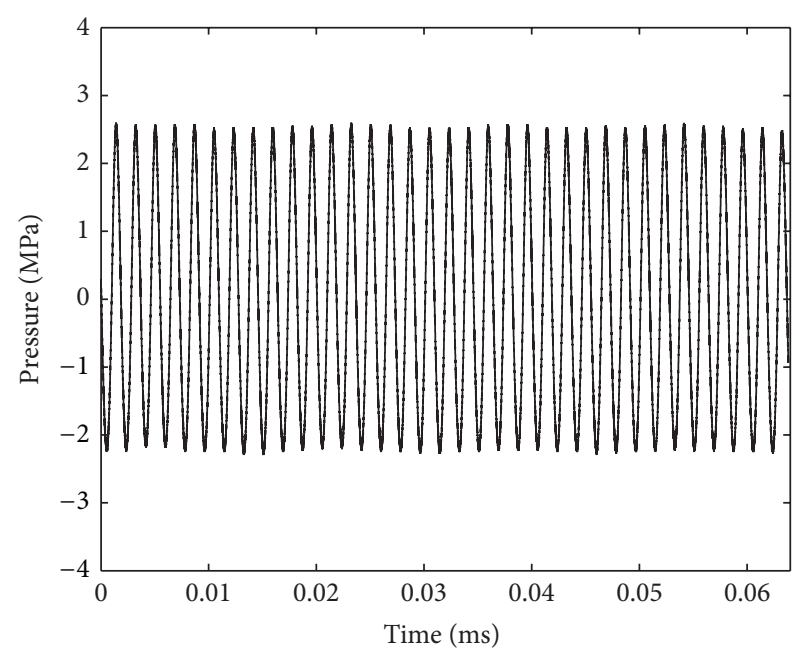

(a)

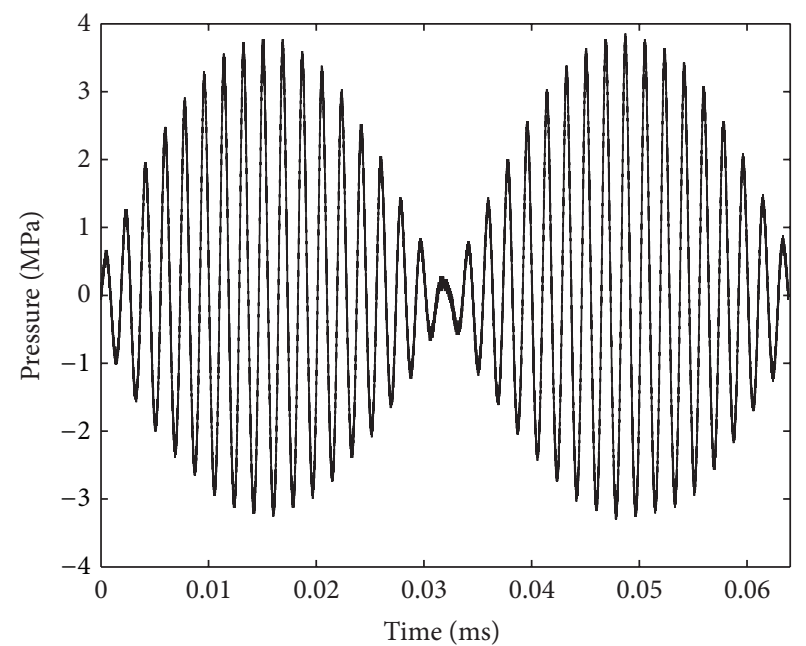

(b)

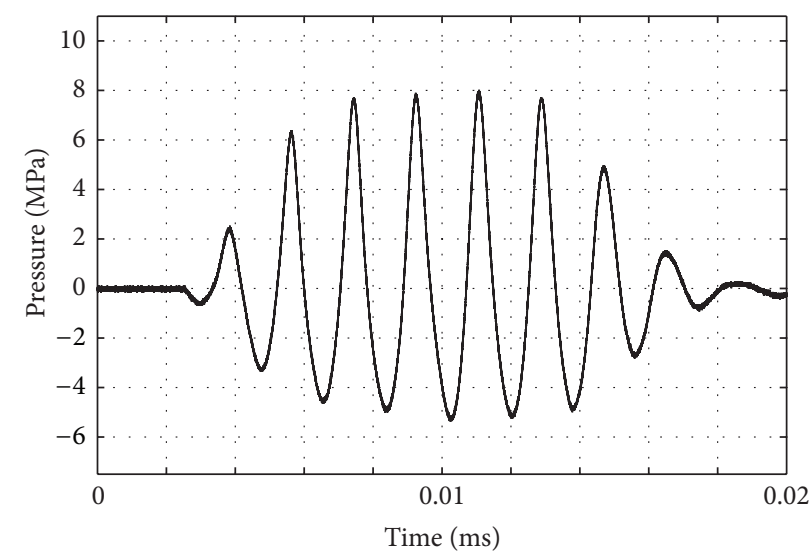

(c)

Figure 1: Pressure waveforms for (a) mono- and (b) bifrequency excitations, measured at the focal point of the transducer at a power $P_{\mathrm{ac}}=$ $25 \mathrm{~W}$. (c) illustrates a pressure waveform measured during the calibration process at $P_{\mathrm{ac}}=165 \mathrm{~W}$. A short pulse was applied to avoid cavitation on the hydrophone. 


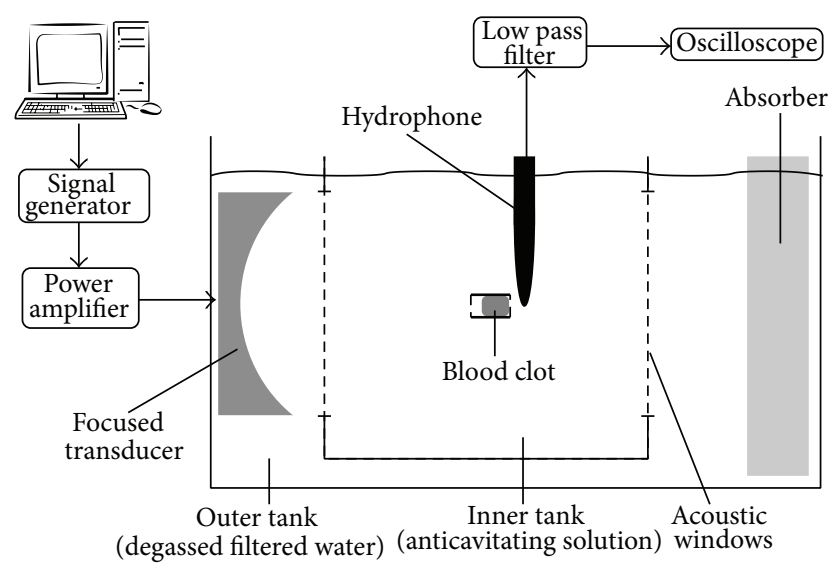

FIGURE 2: Sketch of the experimental setup.

front side of the PVC tube outside from the tube, the smaller tank was filled with a solution of Polyvinylpyrrolidone (PVP) diluted at $30 \mathrm{~g} \cdot \mathrm{L}^{-1}$ [27].

In order to quantify the activity of inertial cavitation during sonication, a low-frequency hydrophone (Reson TC4034, Denmark, bandwidth $[1 ; 500 \mathrm{kHz}]$ ) was placed behind the PVC tube, in the axial plane. The acoustic centre of the hydrophone was positioned $1.2 \mathrm{~cm}$ behind the PVC tube and $1 \mathrm{~cm}$ above the acoustical axis. The signal recorded by the hydrophone was low pass filtered using a 4th order filter with a cut-off frequency of $400 \mathrm{kHz}$, before being digitized using a numerical oscilloscope (Wavesurfer 24XS, Lecroy, USA). For each $300 \mathrm{~s}$ experiment (cf. Section 2.1), 19 signal samples of $27 \mathrm{~ms}$ were saved, one every $16 \mathrm{~s}$. For each sample, the Inertial Cavitation Index (ICI) was defined as the average inertial cavitation activity computed every $1.4 \mathrm{~ms}$ according to the method detailed in [25]. ICI was recorded as a function of time. The ICI values were time-averaged to get $\langle\mathrm{ICI}\rangle$ which was an estimation of the mean inertial cavitation activity during the whole experiment. The plot of thrombolysis efficiency as a function of $\langle\mathrm{ICI}\rangle$ enabled analysing the correlation between cavitation activity and thrombolysis.

Clots were formed from human blood obtained from anonymous, healthy volunteers, under the agreement of the "Etablissement Français du Sang" (French Blood Establishment) and the French Institute of Health and Medical Research (Inserm). Once the blood sample had been taken, it was kept at $4^{\circ} \mathrm{C}$ during the serological control (between $12 \mathrm{~h}$ and $24 \mathrm{~h}$ ). Then, $100 \mathrm{~mL}$ of blood was mixed, at $37^{\circ} \mathrm{C}$, with $4 \mathrm{mM}$ of $\mathrm{CaCl}_{2}$ and $5 \mathrm{UNIH}$ of human thrombin, which was stabilized with a bovine albumin solution (diluted in physiological serum at $1 \%$ ). The mix was poured into a $100 \mathrm{~mL}$ Petri dish $(\varnothing 100 \mathrm{~mm} \times 12 \mathrm{~mm})$, which was incubated at $37^{\circ} \mathrm{C}$ for 5 min during coagulation process. Once coagulated, the blood was preserved at $4^{\circ} \mathrm{C}$ in the closed Petri dish during three days in order to ensure clot retraction [28-31]. It was then used for the experiments within three days.

To carry out thrombolysis experiments, $10 \mathrm{~mm}$ in diameter and $10 \mathrm{~mm}$ in height cylindrical blood clots (volume
$=785 \mathrm{~mm}^{3}$ ) were taken from Petri dish using a die-cutter. Before sonication, a blood clot was rinsed using physiological serum on a $25 \mu \mathrm{m}$ filter and weighed, providing the initial clot mass $m_{\text {clot }}$. The average weight of clots was $724 \mathrm{mg}$ with standard deviation of $120 \mathrm{mg}$. The blood clot was then placed in the PVC tube; the remaining volume was filled with physiological serum and the tube was closed at both ends with latex membranes. For sonication, the tube was then placed at the focus of the transducer, the liquid part being placed in front of the transducer and the clot being in contact with the back of the tube (cf. Figure 2). The focal point of the transducer was placed $8 \mathrm{~mm}$ deep after the front interface of the clot. After sonication, the PVC tube was drained and rinsed on a $25 \mu \mathrm{m}$ filter and the residual mass ( $\left.m_{\text {residual }}\right)$ was weighed.

The thrombolysis efficiency was defined as the percentage of lysed clot:, $\left(1-m_{\text {residual }} / m_{\text {clot }}\right) \times 100$. For each intensity and each type of excitation, 5 to 7 clots were sonicated; the total number of sonicated clots was 134. Throughout the study, nonsonicated clots were weighed before and after the abovementioned procedure and constituted a control group of 18 samples.

The values were summarized as mean. The relationship between both excitations was examined by the MannWhitney test. $P<0.05$ and $P<0.001$ indicated statistically significant $(*)$ and highly significant difference $(* *)$, respectively.

\subsection{Mono- versus Bifrequency Sonication Follow-Up Using} $M R$ Imaging. In order to further characterize the effects associated with both types of excitation, complementary experiments combining sonication with fast MR acquisitions were performed. A MRI compatible version of the setup described in Section 2.2 enabled the use of a gradient echobased sequence ( $\mathrm{T} 2^{*}$-weighted) for two different sets of experiments: (1) dynamical imaging of lysis process operated on large cylindrical specimens of blood clots ( $30 \mathrm{~mm}$ in diameter, $30 \mathrm{~mm}$ in height); (2) a comparison of the temperature profiles induced by both types of excitation using sensitivity of the gradient echo sequence to temperature elevation (with proton resonance frequency shift effect). Passive detection of cavitation using a hydrophone was not available with MR imaging.

All MR imaging experiments were performed on a $3.0 \mathrm{~T}$ clinical MR-scanner (TIM Trio, Siemens, Germany) using a standard 4-channel flex coil for MR signal acquisition. Measurement of temperature elevation during sonication was performed using MR thermometry based on PRFS effect [32]. A segmented gradient-recalled echo-planar imaging (GREEPI) sequence was used to provide PRFS-sensitive images. The main imaging acquisition parameters were $T R=45 \mathrm{~ms}$, $T E=6.3 \mathrm{~ms}$, and voxel size $=0.75 \times 0.75 \times 5 \mathrm{~mm}(256 \times 256$ matrix). The acquisition time per measurement was $5 \mathrm{~s}$ for two orthogonal interleaved slices (centred on the focal point each and aligned with the revolution axis of the transducer). $\mathrm{RF}$ saturation slabs were prescribed laterally from the target in order to reduce the MR effects of HIFU-induced streaming in the water tank. Due to the limitations of the PRFS 
method, temperature profiles could not be measured during thrombolysis within the blood clot, mainly because of the presence of the moving liquid phase in the lysed region of the thrombus around the focal point of the transducer, as the drilled hole was getting filled with water. Beside the local macroscopic motion of MR observed protons, dynamic changes in the bulk magnetic susceptibility are expected in the process of clot dissolution. The comparison of the temperature profiles with monofrequency and bifrequency sonications was therefore performed in ex vivo turkey muscle.

The sample to be sonicated, either blood clot or turkey muscle, was placed in a PVC tube (ID $=30 \mathrm{~mm}$, OD = $32 \mathrm{~mm}$, and $H=70 \mathrm{~mm}$ ) which was closed at both ends with condoms. Muscle samples completely filled the PVC tubes, while tubes containing the blood clots were filled with physiological serum on the side proximal to the transducer. The tube was positioned in order to get the focal point $5 \mathrm{~mm}$ deep after the front interface of the clot. For muscle samples, the focus was positioned at the center of the sample, $35 \mathrm{~mm}$ away from the front interface. The tubes were immersed in a degassed water tank that ensured acoustic coupling and isothermal conditions at specimen edges.

Temperature maps were calculated offline on a sliceper-slice basis using the reference-free method proposed by [33]. This approach was chosen as it intrinsically removes the need of correction for $B_{0}$ drift and phase shift due to surrounding water streaming. Although this streaming is spatially located outside the region of interest (ROI), it induces "ghosting-" like phase shift effects also inside the ROI via the $k$-space contamination, which further prevents the use of time referenced PRFS thermometry. The Dirichlet domain was defined to be circular and as large as possible within the ex vivo tissue $(20 \mathrm{~mm}$ in diameter). Note that increasing the domain size helps in reducing the risk of thermal contamination of its border and thus the subsequent risk of underestimation of the temperature elevation. The voxels from the domain border having an SNR inferior to 15, if any, were removed from calculation and a closed border was regenerated using the iterative harmonic interpolation described in [33]. Baseline MR acquisition without sonication was performed in order to calculate the intrinsic standard deviation of the MR thermometry (as described in [34]). The pixelwise standard deviation was evaluated to be $0.7^{\circ} \mathrm{C}$.

\section{Results}

3.1. Thrombolysis Efficiency. Thrombolysis efficiencies were measured for both types of excitation and for different power values $\left(P_{\mathrm{ac}}\right)$, and the results are summarized in Figure 3. The baseline thrombolysis for the nonsonicated control group of 18 clots was $12 \pm 7 \%$ (mean \pm standard deviation). Therefore we shall consider the ultrasound-induced thrombolysis as efficient only for clot dissolution above $19 \%$. For the monofrequency excitation, no thrombolysis was detected for power values lower than $130 \mathrm{~W}$. Beyond this exposure level, the thrombolysis efficiency increased to achieve a maximum value of $95 \pm 8 \%$ for a power of $200 \mathrm{~W}$.

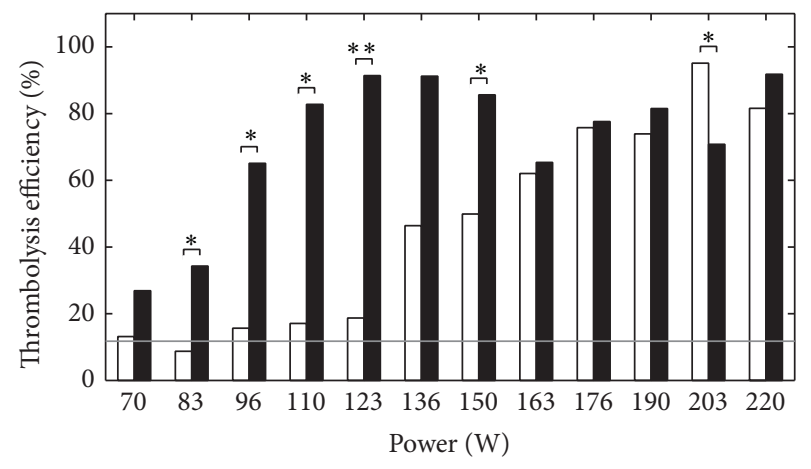

Figure 3: Thrombolysis efficiency as a function of the power $\left(P_{\mathrm{ac}}\right)$, for a monofrequency excitation (white bars) and a bifrequency excitation (black bars). ${ }^{*} P<0.05$ and ${ }^{* *} P<0.001$. The grey line corresponds to the baseline thrombolysis of the nonsonicated control group $(12 \pm 7 \%$ (mean \pm standard deviation) $)$.

In the case of the bifrequency excitation, a thrombolytic effect was observed even for the lowest exposure level $(70 \mathrm{~W})$. The efficiency achieved a maximum value of $91 \pm 10 \%$ at $123 \mathrm{~W}$. For larger exposure levels, the thrombolysis efficiency remained larger than $70 \%$ despite an irregular behavior, mainly due to some screening of the acoustic wave by cavitation bubbles produced upstream the PVC tube at such powers, with a bifrequency excitation.

For powers below $130 \mathrm{~W}$, the bifrequency efficiency was several times larger than the monofrequency one. In particular, at $120 \mathrm{~W}$, the bifrequency efficiency was $90 \%$, while there was no significant thrombolysis with the monofrequency excitation.

3.2. Correlation between Inertial Cavitation Activity and Thrombolysis Efficiency. The thrombolysis efficiency is plotted in Figure 4 as a function of $\langle$ ICI $\rangle$. Despite some dispersion, especially observed for intermediate $\langle\mathrm{ICI}\rangle$ values, one can note an overall increase in thrombolysis efficiency for increasing inertial cavitation activity, with no thrombolysis observed for $\langle$ ICI $\rangle$ below 2000 a.u. For $\langle$ ICI $\rangle$ beyond 6000 a.u., the thrombolysis efficiency was always larger than $50 \%$.

In order to explain the strong dispersion observed for 〈ICI $\rangle$ between 2000 and 6000 a.u., the time fluctuations of ICI have to be considered; Figures 5(a) and 5(b) show the time evolution of ICI for two realizations corresponding to an identical bifrequency treatment at $110 \mathrm{~W}$. In both cases, $\langle\mathrm{ICI}\rangle$ was about 3000 a.u. while the corresponding thrombolysis efficiencies were, respectively, 11\% for Figure 5(a) and 53\% for Figure 5(b). However, ICI time evolutions were very different from one case to the other: in Figure 5(a) ICI was roughly constant during the treatment, whereas it was above 5000 a.u. during the first $50 \mathrm{~s}$ in Figure 5(b). In the latter case, the short duration where ICI > 5000 a.u. appeared to be sufficient to lyse $53 \%$ of the blood clot whereas the constant ICI $\simeq 3000$ a.u. of Figure 5(a) induced no lysis. Similar time behaviours, regardless of the excitation, could be observed for 


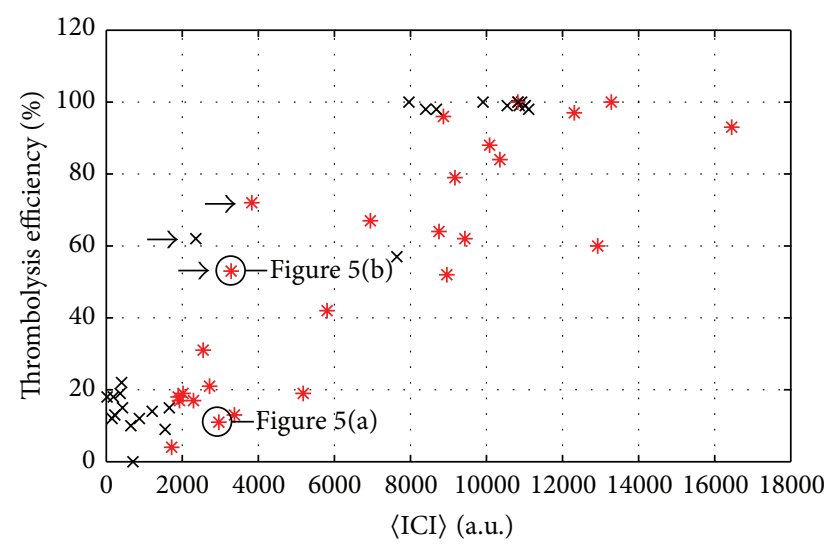

FIgURE 4: Thrombolysis efficiency as a function of average Inertial Cavitation Index $\langle\mathrm{ICI}\rangle$, for a monofrequency excitation (black crosses) and a bifrequency excitation (red asterisks).

the realizations corresponding to the points marked with an arrow in Figure 4.

3.3. MR Monitoring of Mechanical and Thermal Effects. Figure 6 shows the MR magnitude images obtained during sonication of a blood clot sample. The progression of blood clot dissolution during the procedure was clearly visible as an increase in MR signal in the hole drilled by ultrasound, once it was filled with physiological serum.

The same setup, using turkey muscle samples instead of blood clots, enabled the comparison of thermal effects for mono- and bifrequency excitations. Typical temperature profiles, along transverse axis in the focal plane at the end of the sonication, are shown in Figure 7 for a power of $200 \mathrm{~W}$. The profile was averaged over 5 samples for each type of excitation, and the intersample standard deviation, omitted from the graph for clarity reasons, was equal to $3.9^{\circ} \mathrm{C}$ and $4.8^{\circ} \mathrm{C}$ on average over the whole profile for bifrequency and monofrequency excitations, respectively. For any exposure level, the profiles were the same for both types of excitation and the maximal temperature rise was achieved at the focus.

Figure 8 shows the average temperature rise reached in muscle samples at the focus for both types of excitation as a function of the power $\left(P_{\mathrm{ac}}\right)$. Regardless of the type of excitation (mono- or bifrequency) the local heating patterns seen by MR thermometry in ex vivo muscle had very similar behavior as a function of the applied power.

\section{Discussion}

It has been proven that transcutaneous ultrasound alone can induce thrombolysis through the development of cavitation activity both in vitro and in vivo [20-23], and it has been demonstrated that inertial cavitation plays a major role in the process. Nevertheless, optimizing treatment strategies is still needed to avoid undesirable effects such as inducing lesions to surrounding healthy tissue or tearing off large debris during lysis that could induce embolisation.
From that perspective, a better understanding of the mechanisms involved is a key point in the development of such techniques. Our results concerning passive listening of inertial cavitation during in vitro blood clot sonication clearly demonstrated the correlation between inertial cavitation activity and thrombolysis efficiency. But this correlation did not concern the average value over the whole treatment duration. To be efficient for pure ultrasound thrombolysis, the inertial cavitation activity must be quite intense. If the ICI was below 3000 a.u., there was no thrombolysis. Beyond this onset threshold value, the thrombolysis of the clot can be complete in less than $300 \mathrm{~s}$.

In a previous study [25], it was shown that a bifrequency excitation is an efficient means to lower cavitation thresholds when they correspond to high acoustic intensities and to stimulate cavitation activity beyond threshold. In the present study, the results show that the use of a bifrequency excitation drastically enhances thrombolysis efficiency in the intensity range where a monofrequency excitation is poorly efficient. This is notably the case below $120 \mathrm{~W}$, and at this exposure level, the bifrequency thrombolysis efficiency achieved $90 \%$ while there was no significant thrombolysis with the monofrequency excitation. A consequence is that the power needed to obtain $80 \%$ of thrombolysis efficiency can be reduced by half $(200 \mathrm{~W}$ with the monofrequency excitation against $110 \mathrm{~W}$ with the bifrequency excitation).

In [25], the bifrequency excitation was able to lower the inertial cavitation threshold only for spatial-peak pulseaverage $\left(I_{\text {sppa }}\right)$ intensities larger than $100 \mathrm{~W} / \mathrm{cm}^{2}$. It has to be noticed that, in that study, cavitation occurred on a sandpaper target and not on a blood clot: the ratio of the acoustic impedances between water and such targets is much higher than the ratio between water and blood clots; peak pressure at the interface was higher for a given amplitude of the incident wave, resulting in an easier cavitation triggering. As a consequence, in the present study, higher amplitudes had to be used to obtain cavitation: at the lowest exposure level used in this study, the spatial-peak pulse-average intensity reaches $500 \mathrm{~W} / \mathrm{cm}^{2}$ (see Table 1), and no cavitation was observed for the monofrequency excitation. At such intensities, one can expect a strong stimulation of the cavitation activity due to the use of a bifrequency excitation, according to the results observed in [25]. This results in the higher thrombolysis efficiencies observed in Figure 3 when a bifrequency excitation is used.

Concerning MR results, the choice of a high duty cycle value enabled comparing heat deposition for both types of excitations and to better characterize the behaviour of the bifrequency waveforms at high amplitudes in tissue. Heating induced by ultrasound wave for a given intensity appeared to be the same for both types of excitation: spatial temperature profiles and temperature maxima measured using MR thermometry in muscle samples were basically identical at a given applied $P_{\mathrm{ac}}$ value. In fact, the use of bifrequency HIFU to enlarge heating lesions in tissue is valid only for amplitudes higher than the cavitation threshold [35], while the constant slopes observed on curves from Figure 8 suggest that no increase of absorption due to the presence 


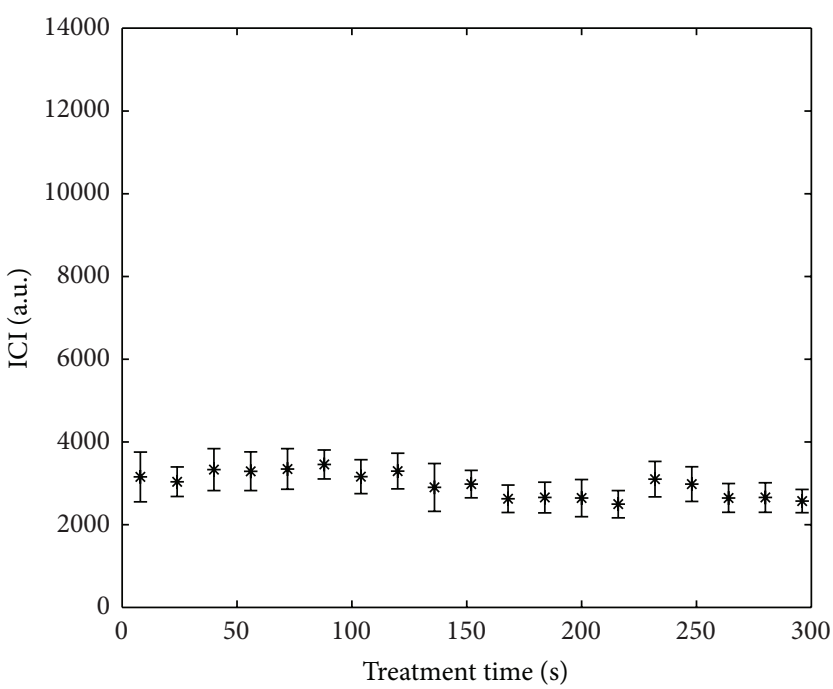

(a)

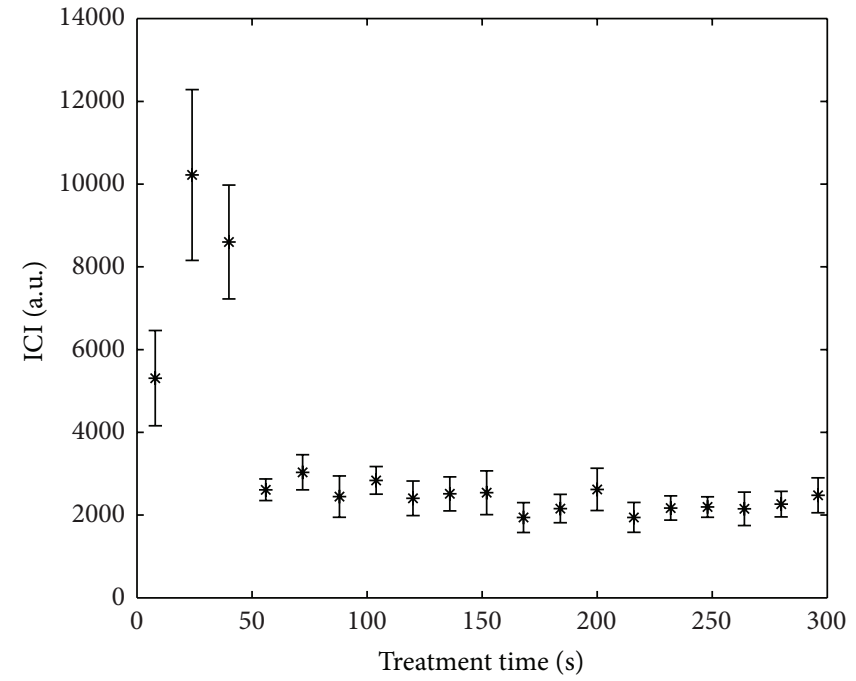

(b)

Figure 5: Examples of time evolution of the Inertial Cavitation Index (ICI) for two samples (bifrequency excitation, $P_{\mathrm{ac}}=110 \mathrm{~W}$ ). Error bars show standard deviation. 〈ICI $\rangle$ on total treatment duration is about 3000 a.u. for both cases. The corresponding thrombolysis efficiency is $11 \%$ for (a) and $53 \%$ for (b).

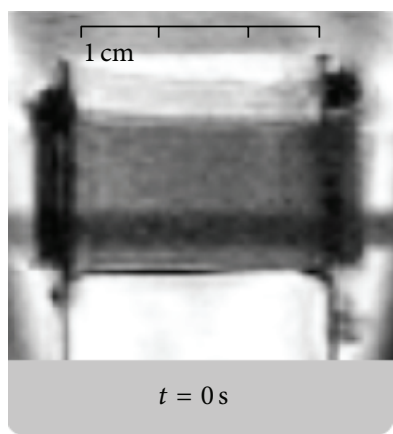

(a)

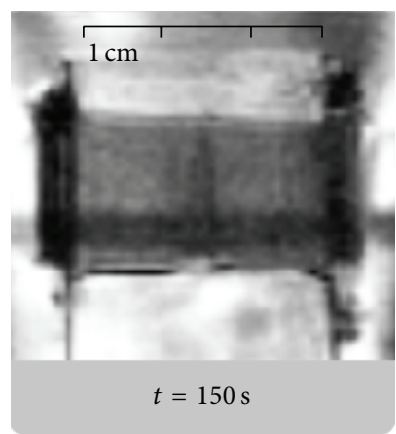

(b)

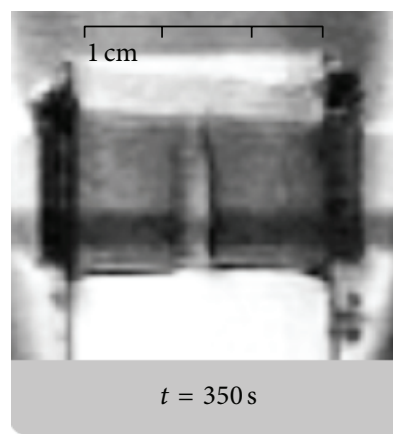

(c)

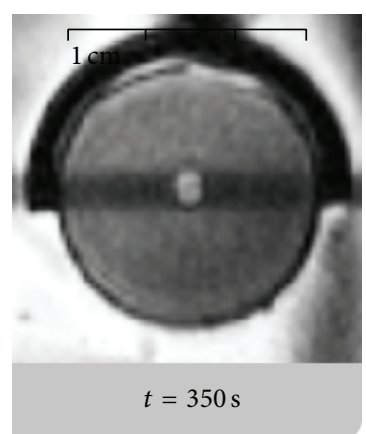

(d)

FIGURE 6: MR imaging of a blood clot during sonolysis (bifrequency excitation, $P_{\mathrm{ac}}=40 \mathrm{~W}$ ) before (a), during (b), and after (c and d) the complete drilling of the sample. ( $\mathrm{a}, \mathrm{b}$, and c) Acquisitions are performed in coronal plane aligned with the acoustical axis while (d) corresponds to a through-focus axial plane (orthogonal to the acoustical axis). The hypointense bands correspond to MR signal saturation at interleaved slice crossing.

of cavitation bubbles occurred during experiments. As for aspects related to the nonlinear propagation of the wave, it was explained in [25] that even for high peak-to-peak amplitude the waveforms should not be shocked because the distance of shock formation is several times higher than the axial length of the $-3 \mathrm{~dB}$ focal volume $(20 \mathrm{~mm})$. The heating patterns obtained in muscle confirmed this aspect and also validated that the acoustic magnitude fields are the same for both types of excitation. For a given acoustic power in liquid, cavitation would be more intense for bifrequency excitation and the nonlinearity would be increased, yielding a larger contribution in terms of nonlinear generation of heat than in structured biological tissue, but for the same thrombolysis efficiency (i.e., the same cavitation activity and the same local nonlinearity due to bubbles) the power required would be lower for bifrequency excitation and so should be the heating.
Obviously, lower duty cycle would be required for in vivo thrombolysis applications, in order to reduce time-average intensities and associated temperature rise to safer values. To evaluate the impact this would have on the thrombolysis efficiency, a comparison of our results in the case of a monofrequency excitation to the one obtained in $[20,22]$, which use 20 to 100 times lower duty cycle values, can be done. Pulse-average acoustic power used in our experiments ranges from 70 to $220 \mathrm{~W}$, with thrombolytic efficiency reaching $50 \%$ at $150 \mathrm{~W}$. For in vitro results presented in [22] acoustic power ranged from 120 to $185 \mathrm{~W}$, with positive results for thrombolysis beyond $160 \mathrm{~W}$. In [20], exposure levels were expressed in terms of peak pressures. Peak negative pressure was ranging from 2 to $12 \mathrm{MPa}$, with thrombolytic effects observed beyond $6 \mathrm{MPa}$. We use 3 to $5.5 \mathrm{MPa}$, with $50 \%$ efficiency achieved at $5 \mathrm{MPa}$. This shows that, when using 


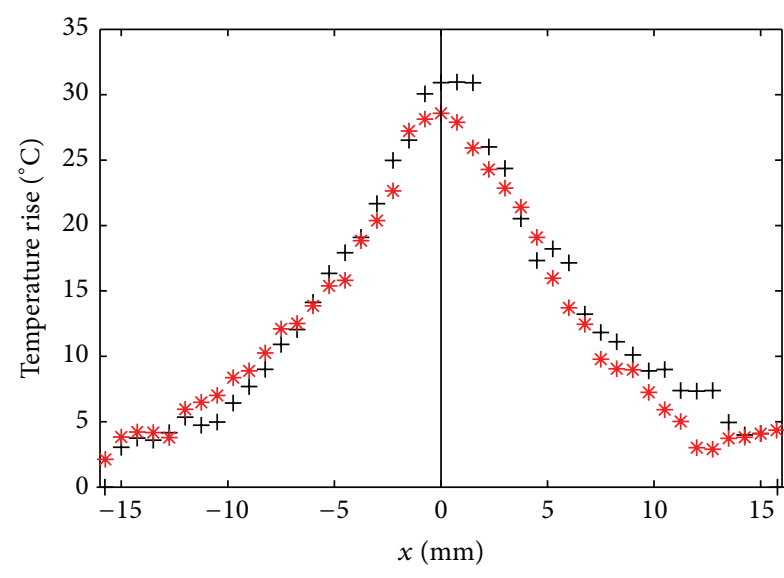

Figure 7: Temperature rise along transverse axis for a monofrequency excitation (black crosses) and a bifrequency excitation (red asterisks), after $300 \mathrm{~s}$ of pulsed sonication $10 \%$ duty cycle. The power is equal to $200 \mathrm{~W}$ for both cases. The origin represents the intersection with the acoustical axis of the transducer.

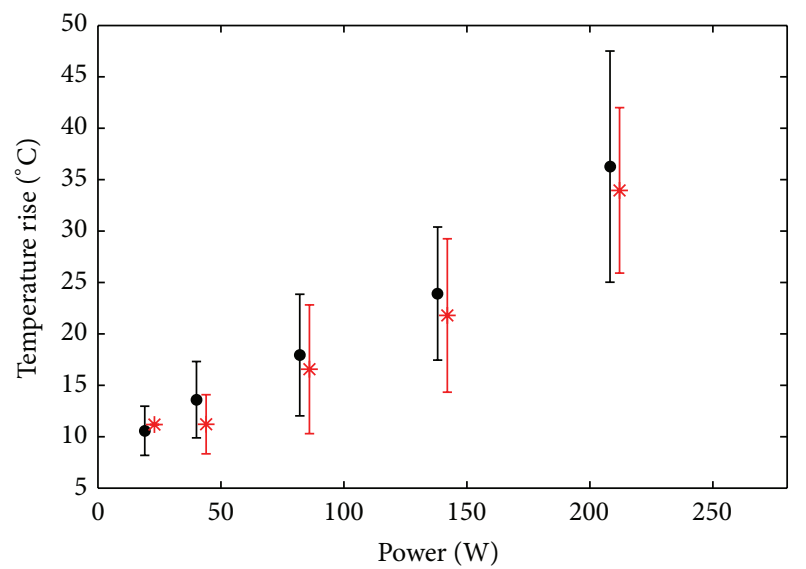

Figure 8: Average temperature rise at the focus as a function of the applied power, for a monofrequency excitation (black discs) and a bifrequency excitation (red asterisks), after $300 \mathrm{~s}$ of pulsed sonication $10 \%$ duty cycle. Error bars show intersample standard deviation.

a monofrequency waveform, we achieve thrombolysis with pulse-average powers or peak negative pressures similar to the one obtained in those studies which use much lower duty cycle values. Reducing the duty cycle should thus reduce the heat deposition without altering our results for thrombolysis efficiency.

It is also worth noting that MR magnitude data enable online visualization of the blood clot dissolution process (see progressive signal modification in the blood clot, near the focal point in Figure 6). This effect could be used for the online monitoring of treatment progress in situ. Nevertheless, a quantitative assessment of the correlation between the variation of MR signal and the structural change in tissue (clot dissolution) is beyond the purpose of this study.

To sum up, the study hereby confirms in vitro the interest of a bifrequency excitation compared with a monofrequency one for pure ultrasound thrombolysis. Taking into account the high values of inertial cavitation threshold of blood [36, 37] and nonlinearity parameter of blood and tissue compared to water [38], the bifrequency excitation should be even more efficient for in vivo thrombolysis than what is observed for the in vitro experiments presented here, since the efficiency of the bifrequency excitation increases with the inertial cavitation threshold of the medium [25].

\section{Conclusions}

The thrombolysis efficiency of a bifrequency excitation consisting in two high neighbouring frequency components has been tested on an in vitro blood clot model and compared to the thrombolysis efficiency of a monofrequency excitation of the same ultrasound intensity. The bifrequency excitation could lower by half the power required to achieve a lysis of a $0.8 \mathrm{~cm}^{3}$ blood clot in 5 minutes. The phenomenon involved in thrombolysis enhancement is confirmed to be inertial cavitation and the thrombolysis efficiency is shown to depend on the whole cavitation activity history: the cavitation activity has to be larger than a given threshold, but once this condition is fulfilled, the lysis is achieved very rapidly. MR thermometry measurements show that the temperature elevation does not depend on the type of excitation (mono- or bifrequency) by itself but only on the acoustic intensity if cavitation is absent. Concerning the therapeutic application, this study shows that the use of a bifrequency excitation is an interesting way to enhance pure ultrasound thrombolysis by stimulating inertial cavitation activity.

\section{Conflict of Interests}

The authors declare that there is no conflict of interests regarding the publication of this paper.

\section{Acknowledgments}

This work was supported by the French National Research Agency (ANR) as part of Project ULysSE ANR-2011-JSV5008-01. The access to MRI facilities was provided by the Center for Biomedical Imaging (CIBM) at University Hospitals of Geneva.

\section{References}

[1] D. W. Barritt and S. C. Jordan, "Anticoagulent drugs in the treatment of pulmonary embolism: a controlled trial," The Lancet, vol. 275, no. 7138, pp. 1309-1312, 1960.

[2] C. Bode, K. Peter, T. Nordt et al., "New developments in thrombolytic therapy," Fibrinolysis and Proteolysis, vol. 11, no. 1, pp. 109-114, 1997.

[3] M. Mehdiratta and L. R. Caplan, "Stroke thrombolysis 2006: an update progress in cardiovascular diseases," Progress in Cardiovascular Diseases, vol. 49, no. 6, pp. 430-438, 2007.

[4] I. A. Khan and R. M. Gowda, "Clinical perspectives and therapeutics of thrombolysis," International Journal of Cardiology, vol. 91, no. 2-3, pp. 115-127, 2003. 
[5] G. J. O’Sullivan, D. G. Lohan, N. Gough, C. G. Cronin, and S. T. Kee, "Pharmacomechanical thrombectomy of acute deep vein thrombosis with the Trellis- 8 isolated thrombolysis catheter," Journal of Vascular and Interventional Radiology, vol. 18, no. 6, pp. 715-724, 2007.

[6] S. Vedantham, "Interventional approaches to deep vein thrombosis," The American Journal of Hematology, vol. 87, no. S1, pp. S113-S118, 2012.

[7] S. Kaul, "Sonothrombolysis: a universally applicable and better way to treat acute myocardial infarction and stroke? Who is going to fund the research?" Circulation, vol. 119, no. 10, pp. 1358-1360, 2009.

[8] W. Steffen, M. C. Fishbein, H. Luo et al., "High intensity, low frequency catheter-delivered ultrasound dissolution of occlusive coronary artery thrombi: an in vitro and in vivo study," Journal of the American College of Cardiology, vol. 24, no. 6, pp. 1571-1579, 1994.

[9] R. D. Shlansky-Goldberg, "Catheter-delivered ultrasound potentiates in vitro thrombolysis," Journal of Vascular and Interventional Radiology, vol. 7, no. 3, pp. 313-320, 1996.

[10] S. Atar, H. Luo, T. Nagai, and R. J. Siegel, "Ultrasonic thrombolysis: catheter-delivered and transcutaneous applications," European Journal of Ultrasound, vol. 9, no. 1, pp. 39-54, 1999.

[11] K. Tachibana, "Enhancement of fibrinolysis with ultrasound energy," Journal of Vascular and Interventional Radiology, vol. 3, no. 2, pp. 299-303, 1992.

[12] H. Luo, T. Nishioka, M. C. Fishbein et al., "Transcutaneous ultrasound augments lysis of arterial thrombi in vivo," Circulation, vol. 94, no. 4, pp. 775-778, 1996.

[13] T. Nishioka, H. Luo, M. C. Fishbein et al., "Dissolution of thrombotic arterial occlusion by high intensity, low frequency ultrasound and dodecafluoropentane emulsion: an in vitro and in vivo study," Journal of the American College of Cardiology, vol. 30, no. 2, pp. 561-568, 1997.

[14] H. Luo, Y. Birnbaum, M. C. Fishbein et al., "Enhancement of thrombolysis in vivo without skin and soft tissue damage by transcutaneous ultrasound," Thrombosis Research, vol. 89, no. 4, pp. 171-177, 1998.

[15] C. W. Francis, "Ultrasound-enhanced thrombolysis," Echocardiography, vol. 18, no. 3, pp. 239-246, 2001.

[16] S. Atar and U. Rosenschein, "Perspectives on the role of ultrasonic devices in thrombolysis," Journal of Thrombosis and Thrombolysis, vol. 17, no. 2, pp. 107-114, 2004.

[17] R. Kornowski, R. S. Meltzer, A. Chernine, Z. Vered, and A. Battler, "Does external ultrasound accelerate thrombolysis? Results from a rabbit model," Circulation, vol. 89, no. 1, pp. 339344,1994

[18] S. Westermark, H. Wiksell, H. Elmqvist, K. Hultenby, and H. Berglund, "Effect of externally applied focused acoustic energy on clot disruption in vitro," Clinical Science, vol. 97, no. 1, pp. 67-71, 1999.

[19] U. Rosenschein, V. Furman, E. Kerner, I. Fabian, J. Bernheim, and Y. Eshel, "Ultrasound imaging-guided noninvasive ultrasound thrombolysis: preclinical results," Circulation, vol. 102, no. 2, pp. 238-245, 2000.

[20] A. D. Maxwell, C. A. Cain, A. P. Duryea, L. Yuan, H. S. Gurm, and $\mathrm{Z}$. Xu, "Noninvasive thrombolysis using pulsed ultrasound cavitation therapy: histotripsy," Ultrasound in Medicine and Biology, vol. 35, no. 12, pp. 1982-1994, 2009.

[21] A. D. Maxwell, G. Owens, H. S. Gurm, K. Ives, D. D. Myers Jr., and $\mathrm{Z}$. Xu, "Noninvasive treatment of deep venous thrombosis using pulsed ultrasound cavitation therapy (histotripsy) in a porcine model," Journal of Vascular and Interventional Radiology, vol. 22, no. 3, pp. 369-377, 2011.

[22] C. Wright, K. Hynynen, and D. Goertz, "In vitro and in vivo high-intensity focused ultrasound thrombolysis," Investigative Radiology, vol. 47, no. 4, pp. 217-225, 2012.

[23] A. Burgess, Y. Huang, A. C. Waspe, M. Ganguly, D. E. Goertz, and K. Hynynen, "High-intensity focused ultrasound (HIFU) for dissolution of clots in a rabbit model of embolic stroke," PLoS ONE, vol. 7, no. 8, Article ID e42311, 2012.

[24] B. Gilles, J. C. Béra, J. L. Mestas, and D. Cathignol, "Reduction of ultrasound inertial cavitation threshold using bifrequency excitation," Applied Physics Letters, vol. 89, no. 9, Article ID 094106, 2006.

[25] I. Saletes, B. Gilles, and J. Béra, "Promoting inertial cavitation by nonlinear frequency mixing in a bifrequency focused ultrasound beam," Ultrasonics, vol. 51, no. 1, pp. 94-101, 2011.

[26] V. A. Khokhlova, M. R. Bailey, J. A. Reed, B. W. Cunitz, P. J. Kaczkowski, and L. A. Crum, "Effects of nonlinear propagation, cavitation, and boiling in lesion formation by high intensity focused ultrasound in a gel phantom," Journal of the Acoustical Society of America, vol. 119, no. 3, pp. 1834-1848, 2006.

[27] E. Blanc and J. Godo, "Milieu de couplage pour ultrasons de puissance," European Patent EP 1038551 B1, 2000.

[28] C. W. Francis and S. Totterman, "Magnetic resonance imaging of deep vein thrombi correlates with response to thrombolytic therapy," Thrombosis and Haemostasis, vol. 73, no. 3, pp. 386391, 1995.

[29] C. K. Holland, S. S. Vaidya, C. C. Coussios, and G. J. Shaw, "Thrombolytic effects of $120-\mathrm{kHz}$ and $1-\mathrm{MHz}$ ultrasound and tissue plasminogen activator on porcine whole blood clots," The Journal of the Acoustical Society of America, vol. 112, p. 2370, 2002.

[30] S. Datta, C. Coussios, L. E. McAdory et al., "Correlation of cavitation with ultrasound enhancement of thrombolysis," Ultrasound in Medicine and Biology, vol. 32, no. 8, pp. 1257-1267, 2006.

[31] C. K. Holland, S. S. Vaidya, S. Datta, C. Coussios, and G. J. Shaw, "Ultrasound-enhanced tissue plasminogen activator thrombolysis in an in vitro porcine clot model," Thrombosis Research, vol. 121, no. 5, pp. 663-673, 2008.

[32] Y. Ishihara, A. Calderon, H. Watanabe et al., "A precise and fast temperature mapping using water proton chemical shift," Magnetic Resonance in Medicine, vol. 34, no. 6, pp. 814-823, 1995.

[33] R. Salomir, M. Viallon, A. Kickhefel et al., "Reference-free PRFS MR-thermometry using near-harmonic 2-D reconstruction of the background phase," IEEE Transactions on Medical Imaging, vol. 31, no. 2, pp. 287-301, 2012.

[34] M. Rata, R. Salomir, R. Umathum et al., "Endoluminal ultrasound applicator with an integrated RF coil for high-resolution magnetic resonance imaging-guided high-intensity contact ultrasound thermotherapy," Physics in Medicine and Biology, vol. 53, no. 22, pp. 6549-6567, 2008.

[35] S. Maruvada, Y. Liu, B. A. Herman, and G. R. Harris, "Evaluation of temperature rise in a tissue mimicking material during HIFU exposure," Journal of Physics: Conference Series, vol. 279, no. 1, Article ID 012010, 2011.

[36] C. K. Holland and R. E. Apfel, "Thresholds for transient cavitation produced by pulsed ultrasound in a controlled nuclei environment," Journal of the Acoustical Society of America, vol. 88, no. 5, pp. 2059-2069, 1990. 
[37] C. X. Deng, Q. Xu, R. E. Apfel, and C. K. Holland, "In vitro measurements of inertial cavitation thresholds in human blood," Ultrasound in Medicine and Biology, vol. 22, no. 7, pp. 939-948, 1996.

[38] P. N. T. Wells, "Ultrasonic imaging of the human body," Reports on Progress in Physics, vol. 62, no. 5, pp. 671-722, 1999. 


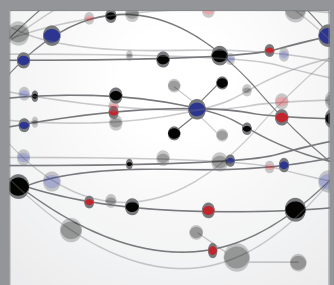

The Scientific World Journal
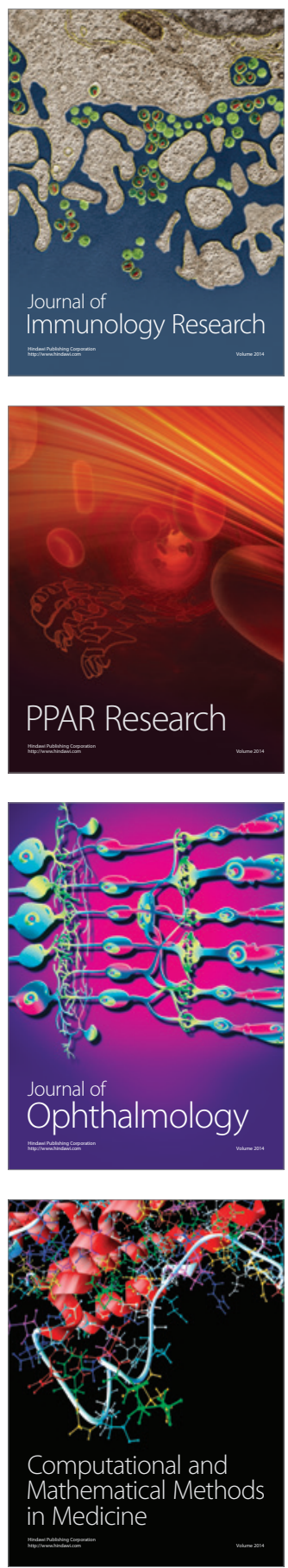

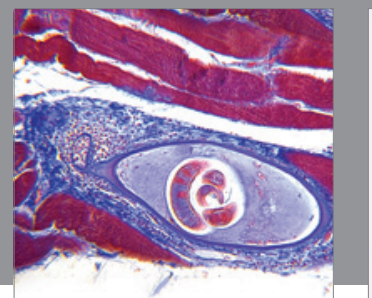

Gastroenterology

Research and Practice
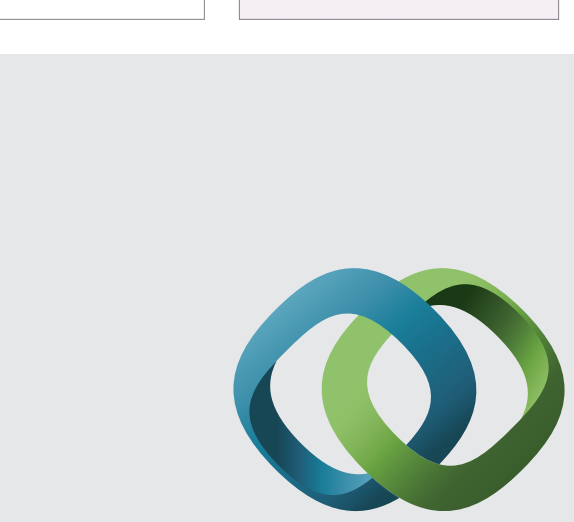

\section{Hindawi}

Submit your manuscripts at

http://www.hindawi.com
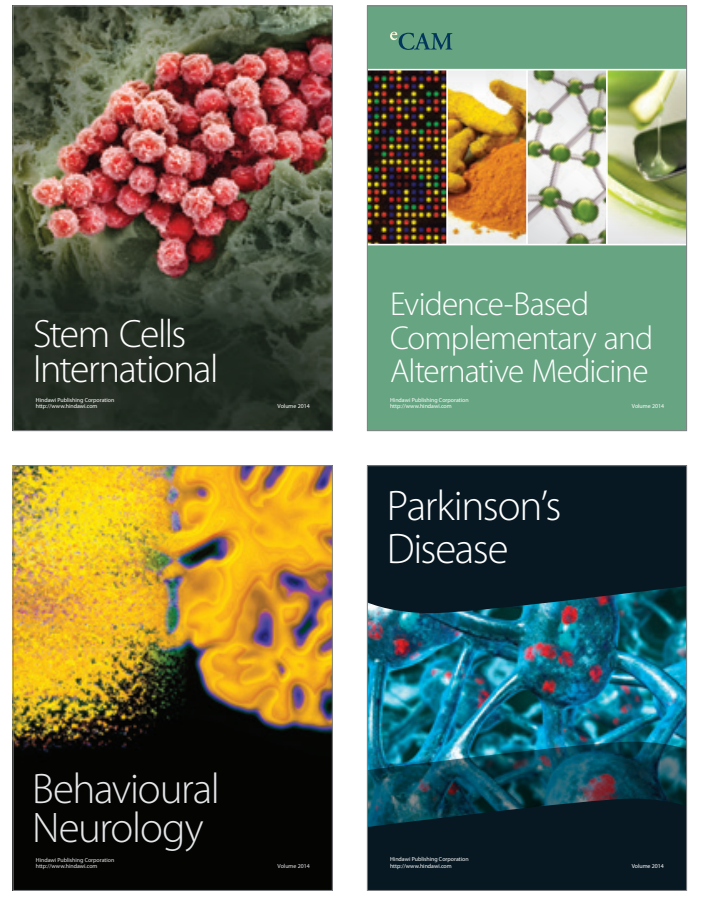
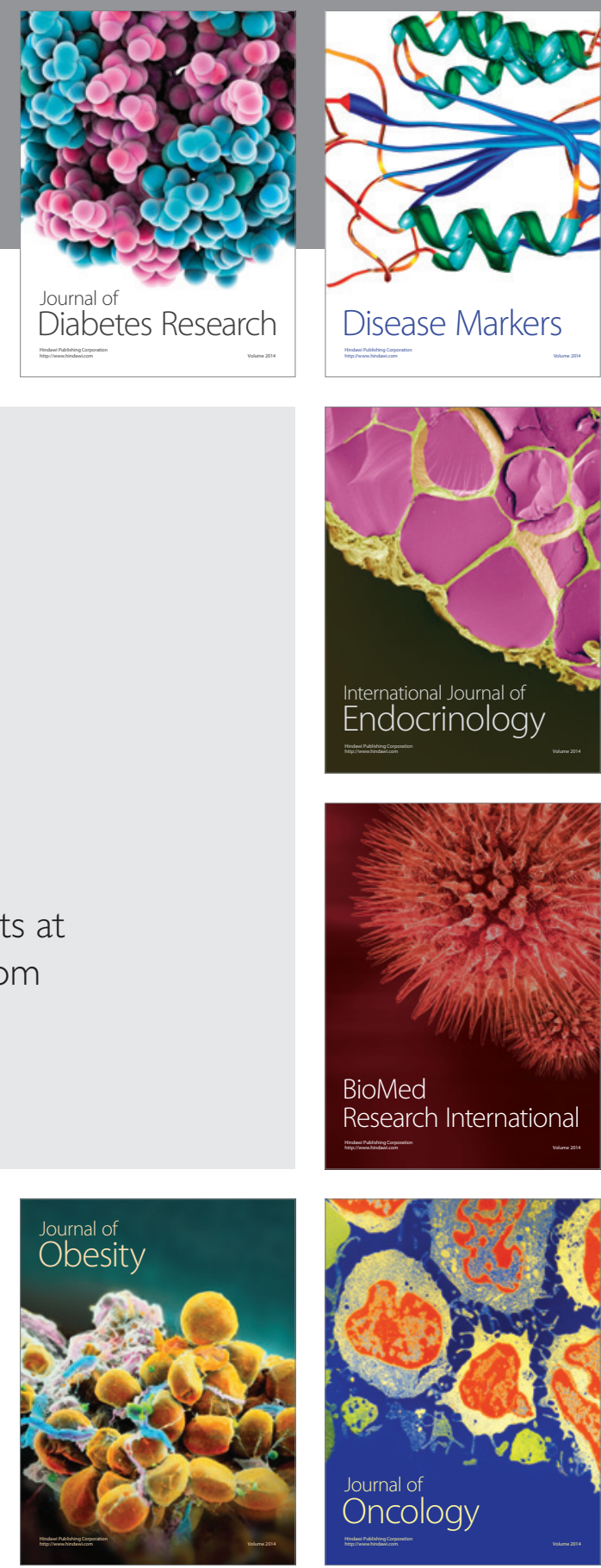

Disease Markers
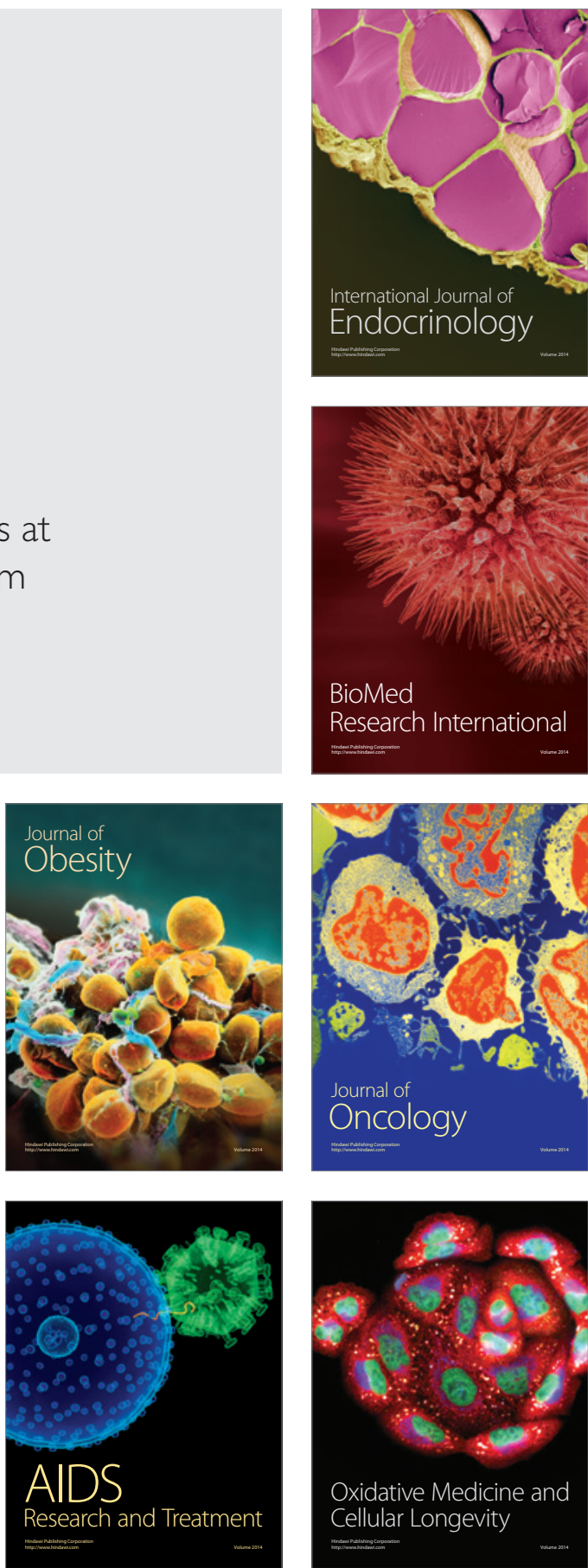